\title{
Intellectual disability and autism: socioeconomic impacts of informal caring, projected to 2030
}

\author{
Deborah Schofield, Melanie J. B. Zeppel, Robert Tanton, J. Lennert Veerman, Simon J. Kelly,
} Megan E. Passey and Rupendra N. Shrestha

\section{Background}

Intellectual disability and autism spectrum disorder (ASD) influence the interactions of a person with their environment and generate economic and socioeconomic costs for the person, their family and society.

\section{Aims}

To estimate costs of lost workforce participation due to informal caring for people with intellectual disability or autism spectrum disorders by estimating lost income to individuals, lost taxation payments to federal government and increased welfare payments.

\section{Method}

We used a microsimulation model based on the Australian Bureau of Statistics' Surveys of Disability, Ageing and Carers (population surveys of people aged 15-64), and projected costs of caring from 2015 in 5-year intervals to 2030.

\section{Results}

The model estimated that informal carers of people with intellectual disability and/or ASD in Australia had aggregated lost income of AU\$310 million, lost taxation of AU\$100 million and increased welfare payments of AU\$204 million in 2015. These are projected to increase to AU $\$ 432$ million, AU\$129 million and AU $\$ 254$ million for income, taxation, and welfare respectively by 2030. The income gap of carers for people with intellectual disability and/or ASD is estimated to increase by 2030 , meaning more financial stress for carers.

\section{Conclusions}

Informal carers of people with intellectual disability and/or ASD experience significant loss of income, leading to increased welfare payments and reduced taxation revenue for governments; these are all projected to increase. Strategic policies supporting informal carers wishing to return to work could improve the financial and psychological impact of having a family member with intellectual disability and/or ASD.

\section{Declaration of interest}

None.

\section{Keywords}

Intellectual disability; autism spectrum disorders; economics; carers; labour force productivity.

\section{Copyright and usage}

(C) The Royal College of Psychiatrists 2019.
Intellectual disability includes a suite of conditions that limit a person's interactions with their environment and incur various economic, psychological and socioeconomic costs to the person, their family and society in general. ${ }^{1}$ Intellectual disability and autism spectrum disorder (ASD) occur in the same person at very high rates. ${ }^{2,3}$ Quality of life (QoL) of people with mild and severe intellectual disability has been estimated at $71 \%$ and $24 \%$ of optimal quality of life respectively, with moderate intellectual disability falling on a spectrum between the two, but these results are based on data from one country only, Australia, and the data are more than 15 years old. ${ }^{4}$ Similarly, the quality of life of individuals with ASD and their families varies according to severity of the disorder. ${ }^{5}$ The costs of caring for people with intellectual disability vary with severity, which is often linked with severe behavioural problems. ${ }^{6}$ Given these quality-of-life and economic factors, people with intellectual disability are a particularly vulnerable group.?

Intellectual disability and/or ASD often reduce the workforce participation of parents or carers ${ }^{5,8,9}$ and it is therefore important to quantify these indirect costs. ${ }^{10}$ Costs of informal care of people with intellectual disability and/or ASD are needed to determine which policies and interventions are most cost-effective for healthcare systems ${ }^{6}$ and, in turn, can best support the return of carers to the workforce. Autism reduces the workforce participation of parental carers, ${ }^{11}$ and it has been reported that $70 \%$ of families caring for children with intellectual disability have reported lost income or reduced workforce hours. ${ }^{6}$ One study found that parents with children younger than 18 with ASD in the USA worked, on average, 7 hours less per week than parents of children without ASD. ${ }^{11}$ A study of children with intellectual disability aged between 5 and 15 years in Australia reported that employment for their parents was difficult because of time demands, unaffordable or inadequate childcare and attitudes of society concerning roles of mothers of children with intellectual disability. ${ }^{8}$

The aims of the present study were to quantify the costs of people being out of the workforce because they are caring for people with intellectual disability and/or ASD. We aimed to (a) estimate the national lost income tax and increased welfare costs, as well as lost income and (b) project the costs of provision of informal care for intellectual disability and/or ASD in 5-year intervals from 2015 to 2030 using counterfactuals to match income for gender, age and educational level, thus reflecting the range of incomes across these variables (this method contrasts with the usual method of using population-average hourly wage multiplied by hours spent caring).

\section{Method}

\section{Data}

We analysed the output data-sets of an Australian microsimulation model, Care\&WorkMOD, designed to project the economic costs of lost productive life years for informal carers aged 15 to 64 for every 5 years from 2015 to 2030. A detailed description of model development is available in Shrestha et al. ${ }^{12}$ Briefly, Care\&WorkMOD was based on unit-record data of individuals aged between 15 and 64 from the Australian Bureau of Statistics' Surveys of Disability, Ageing and Carers (SDACs) in 2003, 2009 and 2012, ${ }^{13-15}$ with additional data from other sources (see below).

The model consists of four main Australian population-representative data-sets: (a) the individual-level data (microdata) on which the model is built; ${ }^{14}$ (b) population and labour force projections from the 2015 Australian Intergenerational Report, which 


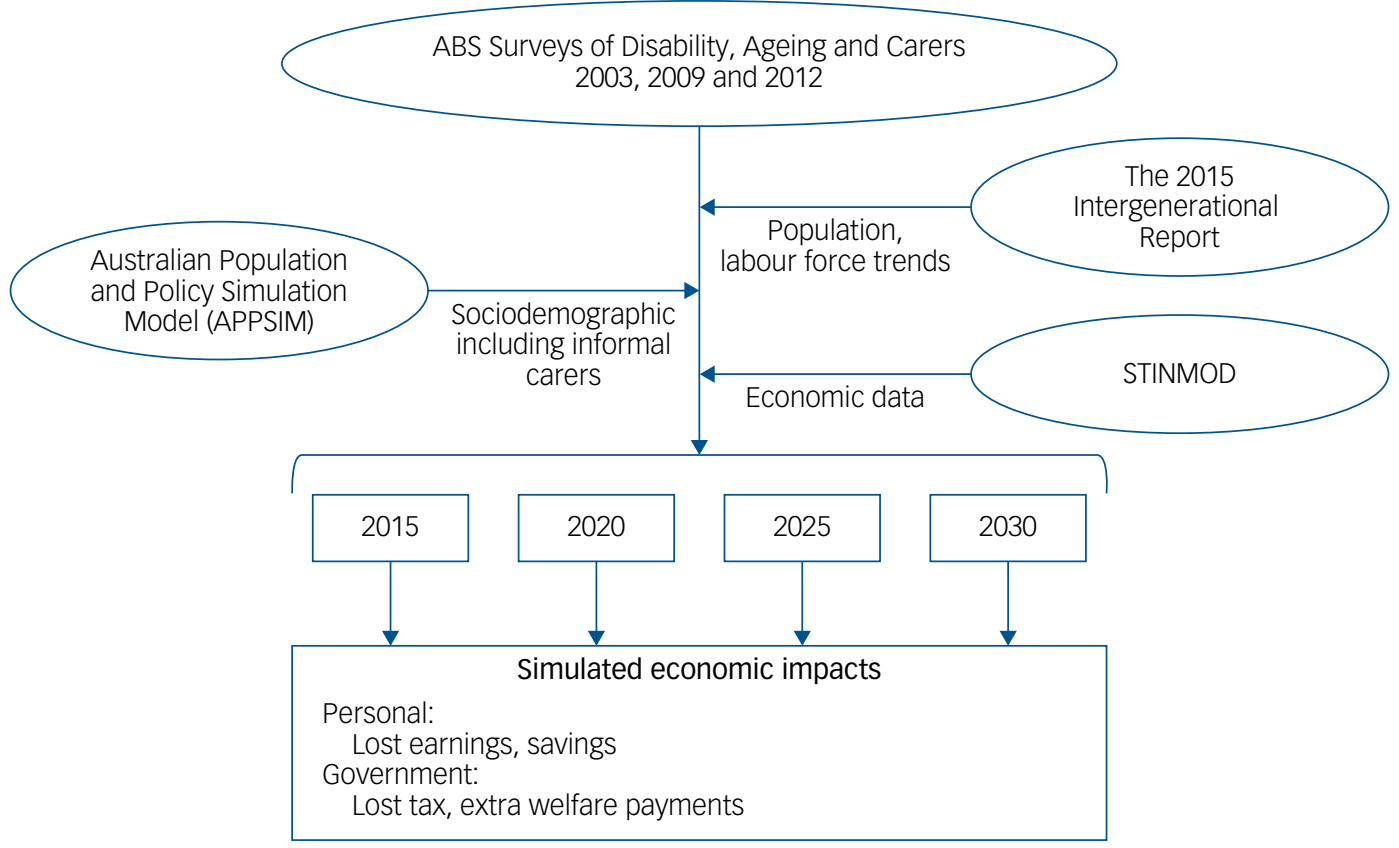

Fig. 1 Input and output models and data comprising the Care\&WorkMOD model. The model includes: data from the Australian Bureau of Statistics' (ABS) Surveys of Disability, Ageing and Carers (SDACS) for 2003, 2009 and 2012; - $\quad$ sociodemographic trends provided by the Australian Population and Policy Simulation Model (APPSIM); economic data provided by the Static Incomes Model (STINMOD); and labour force trends provided by the 2015 Australian Intergenerational Report.

project age and gender distributions; ${ }^{16}$ (c) the projected distributions of sociodemographic variables, including the percentage of informal carers, from the Australian Population and Policy Simulation Model (APPSIM); ${ }^{17}$ and (d) output data-sets from the Static Incomes Model (STINMOD). ${ }^{18}$ These four component data-sets are representative of the Australian population. A schematic diagram of Care\&WorkMOD is provided in Fig. 1.

The model uses static ageing to project the sociodemographic and economic profile of the Australian population to 2015, 2020, 2025 and 2030. Static ageing simulates the population through 'uprating' or 'reweighting'. Uprating is the process of inflating or deflating current monetary values so that they match the projected monetary values. Reweighting is the process whereby the current population distribution, such as the number of people by age group and gender, is changed to match the projected population distribution by altering the weights of each record in the sample. Static ageing does not change the characteristics of individual records in the sample, such as their labour force participation and informal caring status: it only changes the weights to reflect how many people there will be with these characteristics in future.

We reweighted the three SDAC data-sets to account for sociodemographic and economic changes and changes in the number of informal carers between the survey years (2003, 2009 and 2012) and the projection years. We used a reweighting algorithm, called GREGWT, developed at the Australian Bureau of Statistics. ${ }^{19}$ This algorithm uses the generalised regression method to modify the initial survey weights assigned to each SDAC unit record so that the new weighted totals match the externally provided total, such as population, labour force and informal carers projections. This means that the characteristics of each individual record over time do not change, only the weight assigned to each record, i.e. the number of people with these characteristics at a given time point will change.

The additional economic information, such as income, income tax paid and welfare payments from the 2015 STINMOD data-set, was linked to the Care\&WorkMOD base population using synthetic matching. ${ }^{20}$ This creates a synthetic data-set combining information from more that one data-set, where the information is collected from different sources on the same population, but does not have common unique record identifiers for the exact matching of individual records in the data-sets. The process probabilistically matches individual records from more than one data-set using a set of matching variables that are common to both data-sets and strongly related to the main outcome of interest. The nine matching variables that we chose were: labour force status; income unit type; income quintile; gender; age group; hours worked per week; highest level of educational qualification; whether or not a home owner; whether or not receiving Carer Payment (a social security benefit) as matching variables. Income and other economic data from STINMOD were indexed to capture the economic growth projections to 2030. Earnings and taxes paid by individuals were assumed to grow at $1 \%$ per year in real terms, i.e. $1 \%$ above inflation, the same factor as used by the Australian Treasury. ${ }^{21}$ The Age Pension, Disability Support Pension and Carer Payments were assumed to grow at the same rate as earnings (i.e. a real growth of $1 \%$ per year), matching the current government policy on these payments. Other welfare payments were assumed to have no real growth (i.e. assumed to grow at the same rate as inflation), on the basis of the Australian government's policy of increasing welfare payments other than pensions in line with Consumer Price Index growth. $^{21}$

Our analysis focuses on the economic costs of primary carers of individuals with intellectual disability and/or ASD who live with them. We used the 2015 SDAC disease codes, which were equivalent to the ICD-10 categories of 'mental retardation and intellectual disability', 'autism and related disorders (including Rhett's syndrome and Asperger's syndrome)' and 'intellectual and development disorders not elsewhere classified'.

An informal carer is defined in the SDACs, and thus in Care\&WorkMOD, as a person who provides any informal 
assistance, in terms of help or supervision, to someone who has a disability or a long-term health condition, for at least 6 months and with no expectation of monetary compensation. This contrasts with formal carers, who generally receive payment in return and provide these services as their primary occupation and are outside the scope of the SDACs. A primary informal carer is someone who provides the most informal assistance to a person with a disability with one or more of the core activities of mobility, self-care and communication. In the SDACs, respondents were specifically asked whether they were 'informal carers of people with a chronic condition'; those who identified themselves as informal carers were asked to give the range of chronic health conditions their main care recipients had and the main chronic health condition among these. The SDAC also questioned respondents about their labour force status and, if they were out of the labour force, the reason for this. Primary informal carers who reported being out of the labour force because they were caring for 'someone else's ill health or disability' and who reported the intellectual disability and/or ASD disease codes above as the main chronic condition of their main care recipient were considered to be informal carers who were out of the labour force owing to caring in our analysis.

\section{Data availability}

Survey of Disability, Ageing and Caring (SDAC) data are publicly available from the Australian Bureau of Statistics website (www. abs.gov.au/ausstats/abs@.nsf/mf/4430.0). STINMOD is publicly available from the NATSEM website (https://natsem.canberra.edu. $\mathrm{au} /$ models/stinmod/).

\section{Statistical analysis}

This analysis estimated the mean, standard deviation and median weekly income, welfare payments and tax payments for people aged 15 to 64 years who were primary carers and out of the labour force because of their caregiving for people with intellectual disability and/or ASD, and for people who were non-carers and in the labour force (employed full-time, employed part-time). Costs were expressed in real 2015 Australian dollars (AU\$). People out of the labour force because of informal caregiving for people with intellectual disability and/or ASD are hereafter referred to as informal carers' and, in this paper, we are specifically referring only to carers of people with intellectual disability and/or ASD as described in the ICD-10 disease codes discussed above.

We compare the economic outcomes between the three groups: (a) those not in the labour force (or who have lost productive life years) because of their intellectual disability and/or ASD caregiving roles, compared with non-carers in (b) full-time or (c) part-time employment. Differences among these three groups were estimated using counterfactual simulation with Monte Carlo methods. ${ }^{22}$ For each record of those not in the labour force because of their caregiving roles, a counterfactual record was selected at random, with replacement from the pool of non-carers who were in full-time employment; records were matched for age group, gender and highest level of education. The mean of the difference in these economic outcomes between the records of those not in the labour force because of their caregiving roles and their counterfactuals was estimated. Although some studies take the approach of estimating the value of care provided, in this study we focus on actual costs. ${ }^{23}$ A total of 5000 simulations were run, generating 5000 counterfactual data-sets for records of those not in the labour force because of caregiving roles. The average of the 5000 simulations and the $95 \%$ confidence interval (CI), estimated using the percentile method, are reported in this study.

To estimate the economic costs of lost labour force participation of informal carers, counterfactuals were drawn from the pool of non-carers who were in the labour force (i.e. employed full-time or employed part-time or unemployed). Analyses were conducted using SAS, version 9.4 for Windows (SAS Institute, Cary, NC, USA). The use of the data was approved by the Australian Bureau of Statistics Microdata Review Panel and we did not require further ethical approval. No participants were interviewed, as national survey data were used, and therefore participant consent was not required.

\section{Results}

There were 10733 primary carers aged 15 to 64 years providing informal care for someone with intellectual disability or ASD for at least 6 months and living in the same residence as the care recipient, in 2015 (Table 1). Intellectual disability and ASD often occur in the same person $(35 \%$ are estimated to have both conditions and $65 \%$ to have only one), ${ }^{3}$ and in this analysis the numbers of carers of people with intellectual disability and of people with ASD were combined. The numbers of informal carers for someone with intellectual disability and/or ASD ('informal carers') was projected to increase to 13080 in 2030, an increase of 22\%. The proportion of informal carers is, as expected, skewed towards women, with 13$15 \%$ of carers being men and $85-87 \%$ women (Fig. 2).

\section{Weekly income}

Informal carers received a median weekly income that was 38\% that of full-time employed non-carers in 2015 (AU\$484 v. AU\$1280; Table 1). By 2030, informal carers were projected to receive only $35 \%$ of the median income of full-time non-carers (AU\$526 v. AU\$1502). The median total weekly welfare payment was AU $\$ 461$ for informal carers, remaining steady at AU\$461 in 2030. As expected, the median welfare payment for full-time employed non-carers was $\$ 0$. The median total weekly tax payment for informal carers was $\$ 0, A U \$ 254$ less than that for full-time employed non-carers in 2015, a difference that increased to AU\$302 in 2030. The Australian government provides a Carers Allowance and a Carers Payment, meaning that informal carers out of the workforce may have a non-zero income.

\section{Difference in weekly income}

The difference in weekly income, adjusted for age, gender and highest level of education, between full-time employed noncarers and informal carers not in the labour force owing to their caregiving responsibilities was an average of AU $\$ 812$ in 2015, increasing to AU $\$ 950$ in 2030, a $17 \%$ increase (Table 2). The average difference between non-carers employed part-time and informal carers not in the labour force was AU\$257 in 2015, increasing to AU\$293 in 2030, a 14\% increase. The difference in weekly welfare payments received by informal carers not in the labour force and full-time employed non-carers was AU\$411 in 2015 , increasing to AU $\$ 425$ in 2030, a 3\% increase. The average difference in tax paid by full-time non-carers and by informal carers not in the labour force was AU\$258 in 2015, increasing to AU $\$ 283$ in 2030 , a $10 \%$ increase (Table 2). We note that the larger increase in income (17\%) compared with welfare increases (3\%) between 2015 and 2030 means that the income disparity is likely to increase in future generations, leading to compounding disadvantage.

\section{National costs}

The total national loss of individual income due to informal caring was AU\$310 million (95\% CI AU\$255-386 million) in 2015, 


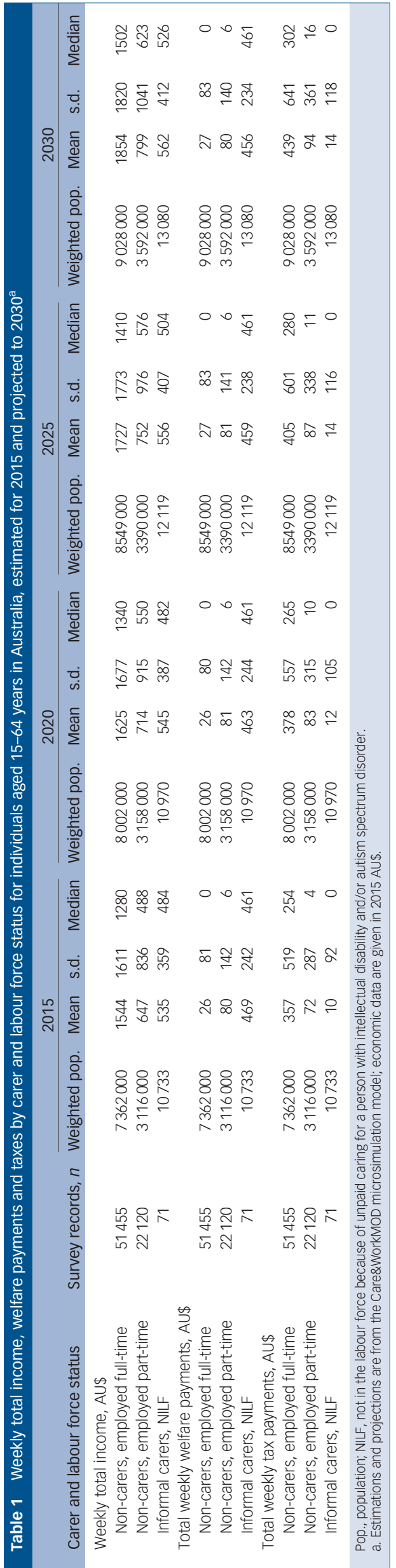

increasing substantially to AU $\$ 432$ million (95\% CI AU\$366-519 million) in 2030 , a $39 \%$ increase. The total cost to the government was projected to grow significantly. Total annual lost taxation revenue to the Australian government due to lost labour force participation of informal carers was estimated at AU $\$ 100$ million in 2015 (95\% CI AU\$82-128 million), increasing to AU\$129 million (95\% CI AU\$107-160 million) in 2030, an increase of 29\%. The total annual cost to the government of extra welfare payments due to informal carers out of the labour force was calculated to be AU\$204 million (95\% CI AU\$192-216 million), rising to AU\$254 million (95\% CI AU\$240-266 million) in 2030, an increase of $24 \%$ (Table 3 ).

\section{Discussion}

Modelling conducted in this study estimated that informal care of people with intellectual disability and/or autism spectrum disorders (ASD) in Australia led to lost income of AU $\$ 310$ million, lost taxation of AU $\$ 100$ million and increased welfare payments of AU\$204 million in 2015. The costs of lost income, lost taxation and increased welfare are projected to rise to AU $\$ 432$ million, AU\$129 million and AU\$254 million respectively in 2030. We note that the increase in lost income compared with the relatively smaller increase in welfare payments means that the income gap between these informal carers and people in the workforce fulltime is likely to increase in future decades, leading to rising financial inequality between informal carers and non-carers. The proportion of women among informal carers for individuals with intellectual disability and/or ASD (85\% women, 15\% men) was much higher than the proportion among informal carers of individuals with back pain (68\% women, $32 \%$ men), another common condition with informal carers out of the workforce. ${ }^{24}$ This is likely due to the earlier age at onset of intellectual disability and/or ASD. Similar to the present study, the proportion of mothers out of the workforce was higher than the proportion fathers out of the workforce in a US study on effects of autism on parental employment. ${ }^{25}$

\section{Caring for individuals with intellectual disability and/or ASD: impact on workforce participation}

This study showed a reduced workforce participation for carers of people with intellectual disability and/or ASD similar to that observed in previous studies from Australia, Japan and the USA. A study of 243 mothers in Japan who were caring for children with intellectual disability showed that they had much a lower employment rate $(49 \%$ compared with $71 \%)$ than mothers of unaffected children of the same age in the same region; $57 \%$ of these unemployed mothers wished to be in the workplace and $62 \%$ of mothers with an intellectually disabled child were in a lower income group. ${ }^{26}$ Single-parent status, good health and college-level education, plus use of childcare and the child's age ( $>12$ years old), were all positively correlated with paid work. ${ }^{26}$ Similarly, a study from the USA found that the impact of having a child with ASD on the parent's workforce participation was influenced by whether the child also had an intellectual disability, and the parent's educational level, immigration status and ethnicity. ${ }^{25}$ An Australian qualitative study reported that, compared with other working Australians, mothers of children with intellectual disabilities had a higher educational level, yet poorer health, lower family income and lower workforce participation. ${ }^{27}$ Furthermore, employment has been reported to improve carers' health, with a study showing that good health was correlated with work and that employment reduced the levels of ill-health of carers of children with intellectual disability. ${ }^{9}$ 


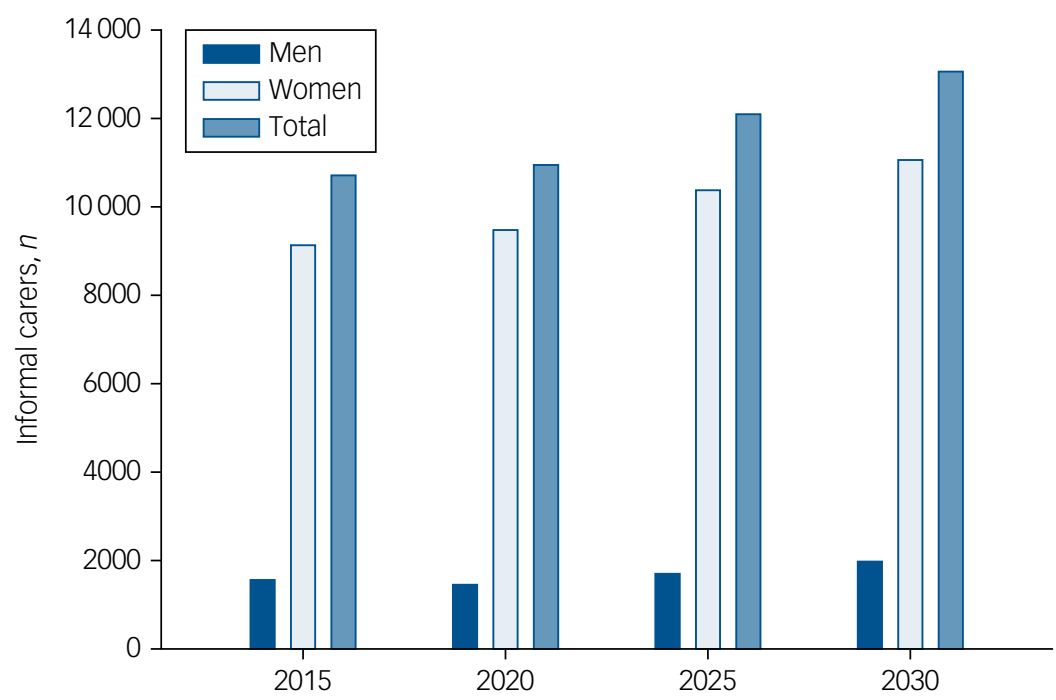

Fig. 2 Projections, by gender, of the number of Australians aged 15-64 years not in the labour force who are informal carers of people with intellectual disability and/or autism spectrum disorder. Data are estimated for 2015 and projected for 2020, 2025 and 2030 on the basis of estimates from the Care\&WorkMOD model described in Fig. 1.

An Australian study from 2012 reported that the majority of families (70\%) caring for children with intellectual disability described lost or reduced income, and that the opportunity cost of lost time is the largest cost to families. ${ }^{6}$ The study surveyed carers and estimated that the time cost of caring for children with mild, moderate or severe intellectual disability was $52 \mathrm{~h}, 61 \mathrm{~h}$ and $85 \mathrm{~h}$ per week respectively. When multiplied by the average wage (AU $\$ 23.67 / \mathrm{h}$ ), used as the cost of care had it been privately funded, the study estimated that the cost of caring for a child with an intellectual disability over 6 months was AU\$31817, AU\$37 786 or AU\$52 494, depending on the severity of the disability. Our study used a different method to estimate the economic impact of caring for intellectual disability: the income reduction due to carers being unable to work, estimated on the basis of 2015 incomes. Our estimate, of AU\$484 for the median weekly income of an informal carer not in the labour force because of caring for intellectual disability and/or ASD, is lower than the AU\$1289 for the average weekly cash earnings of Australian employees. ${ }^{28}$ We took account of the typical female and male labour force patterns, including full-time and part-time work. Our study matched people out of the workforce because of informal caring with people of comparable age, gender and educational levels working full time. Both studies show that the time and lost workforce participation of informal carers is significant, and targeted strategies may be useful to increase their workforce participation.

\section{Informal care influences other parts of the carer's life}

High levels of stress are reported in carers of people with intellectual disability, but employment can give greater empowerment, with higher quality of life and reduced stress. ${ }^{9,27}$ An Australian study of mothers of children with intellectual disability reported that working mothers had significantly better health-related quality of life (HRQoL) than non-working mothers on five of eight dimensions of the 36-item Short Form Health Survey (SF-36). ${ }^{27}$ Similarly, having a job was reported to increase resilience for parents of children with intellectual disability. A study of 32 family carers of people with intellectual disability in Spain also reported that having an occupation gave greater resilience to carers. ${ }^{29}$ In addition to reduced income, lack of opportunities to work leads to feelings of isolation, lack of fulfilment and little release from the pressures of caregiving. ${ }^{8}$ Parents and carers of people with intellectual disability and/or ASD experience a range of financial and psychological problems that need addressing to prevent social inequalities in those families. Intellectual disabilities have been reported to account for and to increase social inequalities,

\begin{tabular}{|c|c|c|c|c|c|c|c|c|}
\hline \multirow[b]{2}{*}{ Carer and labour force status } & \multicolumn{2}{|c|}{2015} & \multicolumn{2}{|c|}{2020} & \multicolumn{2}{|c|}{2025} & \multicolumn{2}{|c|}{2030} \\
\hline & $\begin{array}{c}\text { Difference, } \\
\text { AU\$ }\end{array}$ & $95 \% \mathrm{Cl}$ & $\begin{array}{c}\text { Difference, } \\
\text { AU\$ }\end{array}$ & $95 \% \mathrm{Cl}$ & $\begin{array}{c}\text { Difference, } \\
\text { AU\$ }\end{array}$ & $95 \% \mathrm{Cl}$ & $\begin{array}{c}\text { Difference, } \\
\text { AU\$ }\end{array}$ & $95 \% \mathrm{Cl}$ \\
\hline \multicolumn{9}{|l|}{ Weekly total income, AU\$ } \\
\hline Non-carers employed full-time $v$. carers NILF & 812 & 717 to 928 & 854 & 758 to 970 & 899 & 800 to 1029 & 950 & 849 to 1074 \\
\hline Non-carers employed part-time $v$. carers NILF & 257 & 189 to 367 & 276 & 196 to 390 & 281 & 208 to 395 & 293 & 224 to 395 \\
\hline \multicolumn{9}{|l|}{ Total weekly welfare payments, AU\$ } \\
\hline Non-carers employed full-time $v$. carers NILF & -411 & -395 to -426 & -415 & -398 to -429 & -418 & -402 to 432 & -425 & -410 to -439 \\
\hline Non-carers employed part-time $v$. carers NILF & -342 & -318 to -366 & -346 & -323 to -369 & -349 & -326 to -372 & -357 & -334 to -378 \\
\hline \multicolumn{9}{|l|}{ Total weekly tax payments, AU\$ } \\
\hline Non-carers employed full-time $v$. carers NILF & 258 & 225 to 304 & 265 & 232 to 312 & 273 & 239 to 322 & 283 & 248 to 331 \\
\hline Non-carers employed part-time $v$. carers NILF & 81 & 58 to 118 & 82 & 58 to 121 & 79 & 58 to 115 & 77 & 58 to 107 \\
\hline
\end{tabular}


Table 3 National costs due to lost labour force participation of primary carers (aged 15-64 years) of people with intellectual disability and/or autism spectrum disorder in Australia, estimated for 2015 and projected to $2030^{\text {a }}$

\begin{tabular}{|c|c|c|c|c|c|c|c|c|}
\hline \multirow[b]{2}{*}{ Cost } & \multicolumn{2}{|c|}{2015} & \multicolumn{2}{|c|}{2020} & \multicolumn{2}{|c|}{2025} & \multicolumn{2}{|c|}{2030} \\
\hline & AU\$ million & $95 \% \mathrm{Cl}$ & AU\$ million & $95 \% \mathrm{Cl}$ & AU\$ million & $95 \% \mathrm{Cl}$ & AU\$ million & $95 \% \mathrm{Cl}$ \\
\hline Individuals: lost income & 310 & $255-386$ & 331 & $271-403$ & 382 & $317-464$ & 432 & $366-519$ \\
\hline Government: lost tax payments & 100 & $82-128$ & 103 & $85-132$ & 116 & $95-148$ & 129 & $107-160$ \\
\hline Government: increased welfare payments & 204 & $192-216$ & 209 & $197-220$ & 231 & $218-244$ & 254 & $240-266$ \\
\hline
\end{tabular}

with a significant association between poverty and the prevalence of intellectual disorders. ${ }^{30}$ Thus, to prevent increasing social inequalities, it is important that interventions and policies are implemented that support carers of people with intellectual disability and/or ASD.

\section{Limitations}

A number of limitations exist in the study. First, the data are from the Surveys of Disability, Ageing and Carers (SDACs), which are self-reported by carers. Whether the reference child would meet clinical diagnostic criteria is not known, yet this is common of economic survey reports on families with ASD ${ }^{25}$ Second, the survey data are taken from a subset of the population, although the dataset has been weighted by age, gender and educational level to reflect the general Australia population. Third, it is not always possible to separate costs associated with one disorder, from those associated with other health conditions or impairments. This problem is common when estimating effects of a disorder with comorbidities, such as intellectual disability or autism. ${ }^{3}$ However, these two conditions are commonly linked and occur in the same person, with 35\% of individuals with ASD also having an intellectual disability. ${ }^{25}$

\section{Implications}

Policies

The majority of constraints on workforce participation for carers of people with intellectual disability and/or ASD are related to limitations in support services. ${ }^{27}$ Of 152 mothers surveyed in one study, $82 \%$ wanted and needed paid work but reasons preventing work were dominated by service limitations. ${ }^{4}$ Similarly, a study of 18 mothers of children with intellectual disability aged 5 to 15 reported that employment was difficult owing to time demands, inadequate/ unaffordable childcare and attitudes of society concerning roles of mothers. ${ }^{8}$ In terms of increasing workforce participation, policies addressing services to allow informal carers to return to work may include appropriate and affordable care and flexible workplaces. ${ }^{31}$ To ensure equality among services provided to carers, resources and services devoted to aiding carers of people with intellectual disability or ASD need to be distributed in a rational, equitable and efficient manner. ${ }^{6}$

\section{Equitable distribution of resources}

Multiple studies reported that informal care of people with intellectual disability or ASD is linked to income inequality. ${ }^{32,33}$ One study from South-East Asia reported that resources were not allocated equally in areas with the most need, that is, parents in high-income areas received more services than people in lower-income areas. ${ }^{32}$ This may be due to parents' awareness of service and supports, as well as time constraints and language barriers, and it is worth noting that this study was conducted two decades ago. In addition, studies from Asia and the UK report that more resources are often allocated to areas that are not the areas with the highest level of need. ${ }^{32,33}$ A study from England of informal carers of people with intellectual disability reported that low socioeconomic areas served more patients, yet with the same amount of resources per 100000 of population. ${ }^{33}$ Furthermore, a study from the USA on parents of individuals with ASD reported that reduced hours of market work were more likely among lower-income parents and single-parent households unable to afford childcare, which was more expensive than for children without disabilities or not available at all. ${ }^{25}$ One consequence is that the earnings loss associated with parenting a child with ASD is largest, in terms of percentage, for the poorest families. Taken together with the lower resources available to informal carers in families of lower socioeconomic status, it is important that interventions and policies on resource distribution address economic inequalities. These interventions and policies will benefit society in many ways, including reducing productivity losses, increasing tax revenue and reducing treatment costs. 31,34

People with intellectual disability and ASD are living longer, ${ }^{11}$ and premature babies, born before 32 weeks' gestation, are increasingly surviving, but often with intellectual disability. Similarly, there is an increase in people being diagnosed with ASD. ${ }^{35}$ This confluence of factors means that the number of diagnoses and people living with intellectual disability and/or ASD is increasing, ${ }^{1,3}$ and is likely to increase in future decades. Thus, it is important to know the economic costs of informal care for intellectual disability and/or ASD.

Deborah Schofield, Professor of Health Economics, Centre for Economic Impacts of Genomic Medicine, Department of Economics, Faculty of Business and Economics, Macquarie University, Australia; Melanie J.B. Zeppel (D), Senior Research Fellow, Centre for Economic Impacts of Genomic Medicine, Department of Economics, Faculty of Business and Economics, Macquarie University, Australia; Robert Tanton, Professor National Centre for Social and Economic Modelling University of Canberra, Australia; J Lennert Veerman (P. Professor, School of Medicine, Giffith University, Australia; Simon J. Kelly, Professor, National Centre for Social and Economic Modelling, University of Canberra, Australia; Megan E. Passey, Associate Professor, University Centre for Rural Health, University of Sydney, Australia; Rupendra N. Shrestha, Senior Research Fellow, Centre for Economic Impacts of Genomic Medicine, Department of Economics, Faculty of Business and Economics, Macquarie University, Australia

Correspondence: Rupendra N. Shrestha, Centre for Economic Impacts of Genomic Medicine, 4 Eastern Road, Macquarie University, North Ryde, NSW 2109, Australia. Email: rupendra.shrestha@mq.edu.au

First received 3 Apr 2019, final revision 30 Jul 2019, accepted 16 Aug 2019 
2 Matson JL, Shoemaker M. Intellectual disability and its relationship to autism spectrum disorders. Res Dev Disabil 2009; 30: 1107-14.

3 Srivastava AK, Schwartz CE. Intellectual disability and autism spectrum disorders: causal genes and molecular mechanisms. Neurosci Biobehav Rev 2014; 46(pt 2): 161-74.

4 Begg S, Voss T, Barker B, Stevenson C, Stanley I, Lopez AD. The Burden of Disease and Injury in Australia 2003. Australian Institute of Health and Welfare 2007.

5 Knapp M, Romeo R, Beecham J. Economic cost of autism in the UK. Autism 2009; 13: 317-36.

6 Doran CM, Einfeld SL, Madden RH, Otim M, Horstead SK, Ellis LA, et al. How much does intellectual disability really cost? First estimates for Australia. J Intellect Dev Disabil 2012; 37: 42-9.

7 Flynn S. Perspectives on austerity: the impact of the economic recession on intellectually disabled children. Disabil Soc 2017; 32: 678-700.

8 Shearn J, Todd S. Maternal employment and family responsibilities: the perspectives of mothers of children with intellectual disabilities. J Appl Res Intellect Disabil 2000; 13: 109-31.

9 Gordon M, Rosenman L, Cuskelly M. Constrained labour: maternal employment when children have disabilities. J Appl Res Intellect Disabil 2007; 20 236-46.

10 Schofield D, Zeppel M, Tan O, Lymer S, Cunich MM, Brief SRA. Global history of microsimulation models in health: past applications. Lessons learned and future directions. Int J Microsimulation 2018; 11: 97-142.

11 Buescher AVS, Cidav Z, Knapp M, Mandell DS. Costs of autism spectrum disorders in the United Kingdom and the United States. JAMA Pediatr 2014; 168(8): 721-8.

12 Shrestha R, Schofield D, Zeppel MJB, Cunich MM, Tanton R, Kelly SJ, et al. Care\&WorkMOD: an Australian microsimulation model projecting the economic impacts of early retirement in informal carers. Int J Microsimulation 2018; 11: 78-99.

13 Australian Bureau of Statistics. Information Paper. Basic Confidentialised Unit Record File: Survey of Disability, Ageing and Carers. 2003 (Reissue). Australian Bureau of Statistics, 2005

14 Australian Bureau of Statistics. Survey of Disability, Ageing and Carers: 2009. Australian Bureau of Statistics, 2012.

15 Schofield DJ, Shrestha RN, Percival R, Kelly SJ, Passey ME, Callander EJ. Quantifying the effect of early retirement on the wealth of individuals with depression or other mental illness. Br J Psychiatry 2011; 198: 123-8.

16 Treasury, Commonwealth of Australia. 2015 Intergenerational Report: Australia in 2055. Commonwealth of Australia, 2015.

17 Keegan M, Kelly S. APPSIM - Dynamic Microsimulation Modelling of Social Security and Taxation. National Centre for Social and Economic Modelling University of Canberra, 2009.

18 Percival R, Abello A, Vu Q. STINMOD (Static Income Model). In Modelling Our Future: Population Ageing, Health and Aged Care (eds A Gupta, A Harding). Elsevier, 2007.
19 Bell P. GREGWT and TABLE Macros - Users Guide. Australian Bureau of Statistics, 2000.

20 Rässler S. Statistical Matching: A Frequentist Theory, Practical Applications, and Alternative Bayesian Approaches. Springer-Verlag, 2002.

21 Treasury, Commonwealth of Australia. Budget Paper No 1: Budget Strategy and Outlook 2015-16. Commonwealth of Australia, 2015.

22 Schofield DJ, Shrestha RN, Cunich M, Tanton R, Kelly S, Passey ME, et al. Lost productive life years caused by chronic conditions in Australians aged 45-64 years, 2010-2030. Med J Aust 2015; 203(6): 260.e1-6.

23 Casey B. The Value and Costs of Informal Care. Institute for Employment Research, University of Warwick, 2011.

24 Schofield D, Shrestha RN, Zeppel MJB, Cunich MM, Tanton R, Veerman JL, et al. Economic costs of informal care for people with chronic diseases in the community: lost income, extra welfare payments, and reduced taxes in Australia in 2015-2030. Health Soc Care Community 2018; 27(2): 493-501.

25 McCall BP. Starr EM. Effects of autism spectrum disorder on parental employment in the United States: evidence from the National Health Interview Survey. Commun Work Fam 2018; 21: 367-92.

26 Ejiri K, Matsuzawa A. Factors associated with employment of mothers caring for children with intellectual disabilities. Int J Dev Disabil 2019: 65(4): 239-47.

27 Bourke-Taylor H, Howie L, Law M. Barriers to maternal workforce participation and relationship between paid work and health. J Intellect Disabil Res 2011; 55: $511-20$

28 Australian Bureau of Statistics. 6306.0 - Employee Earnings and Hours, Australia, May 2018. Australian Bureau of Statistics, 2018 (https://www.abs. gov.au/AUSSTATS/abs@.nsf/Lookup/6306.0Main+Features1May\%202018? OpenDocument).

29 Oñate L, Calvete E. A qualitative approach to the resilience factors in families of people with intellectual disabilities in Spain. Psychosoc Interv 2017; 26: 93-101.

30 Emerson E. Poverty and people with intellectual disabilities. Ment Retard Dev Disabil Res Rev 2007; 13: 107-13.

31 Carers NSW. Carers NSW 2018 Carer Survey: Summary Report. Carers NSW, 2018.

32 Hatton C, Azmi S, Caine A, Emerson E. Informal carers of adolescents and adults with learning difficulties from the South Asian communities: family circumstances, service support and carer stress. Brit J Soc Work 1998; 28: 821-37.

33 Chinn D, Levitan T, Murrells T. Equity in social care for people with intellectual disabilities? A cross-sectional study examining the distribution of social care funding across local authorities in England. Health Soc Care Community 2017; 25: 901-11.

34 Marmot M, Allen J, Goldblatt P, Boyce T, McNeish D, Grady M, et al. Fair Society, Healthy Lives: The Marmot Review. Strategic Review of Health Inequalities in England Post-2010. The Marmot Review, 2010 (http://www.instituteofhealthequity.org/resources-reports/fair-society-healthy-lives-the-marmotreview/fair-society-healthy-lives-full-report-pdf.pdf).

35 Dansby RA, Turns B, Whiting JB, Crane J. A phenomenological content analysis of online support seeking by siblings of people with autism. J Fam Psychother 2018; 29: 181-200. 